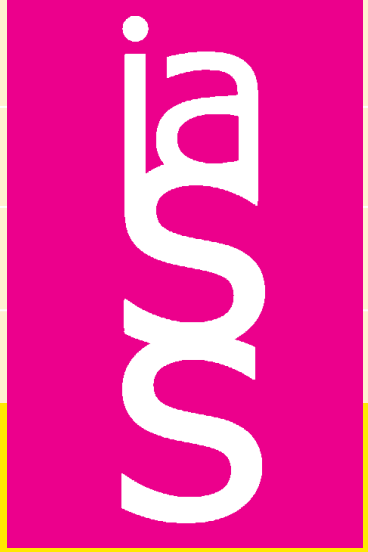

JOURNAL OF

THE INTERNATIONAL ASSOCIATION

FOR SHELL AND SPATIAL

STRUCTURES

Prof. D. h-C Eng. E. TORROJA, founder

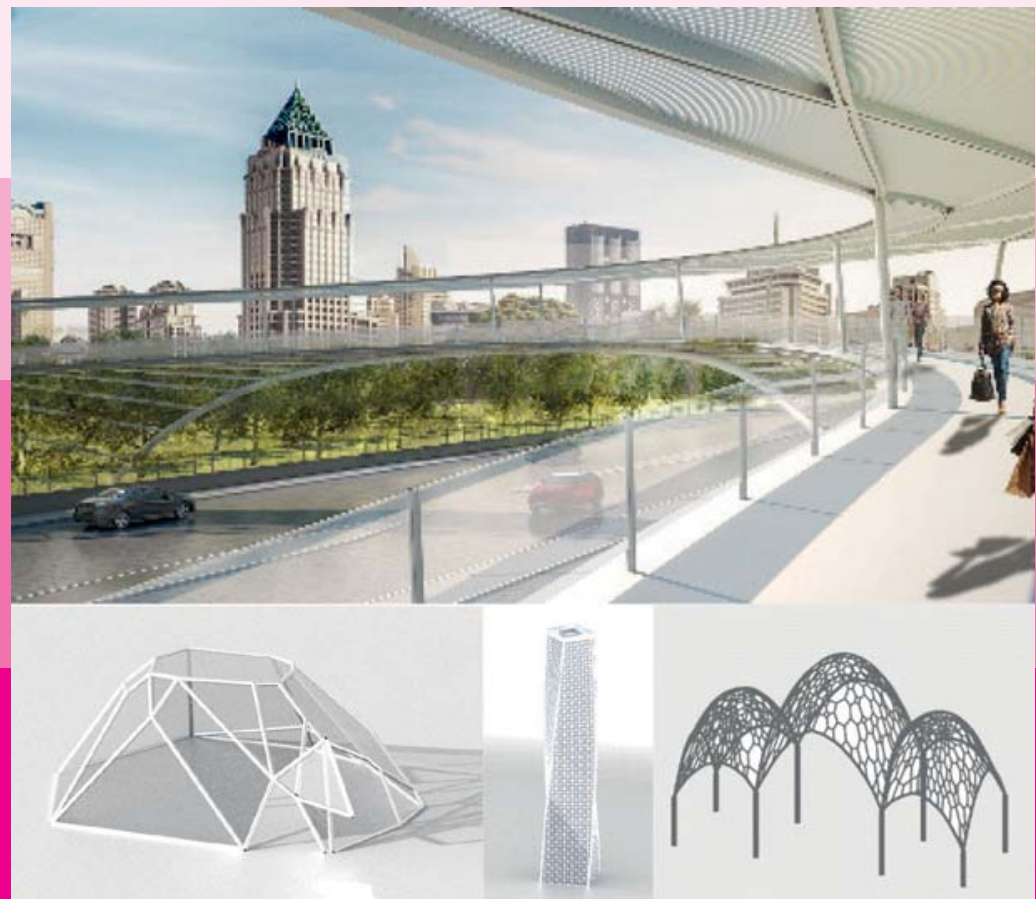

Vol. 59 (2018) No. 4

December n. 198

ISSN: 1028-365X 


\section{n. 198 December}

Annual Letter from the President

S. Pellegrino

\section{Announcements}

IASS Symposium Announcement 2019

Tsuboi Proceedings Award Paper for 2017

The Application of 2D and 3D Graphic Statics in Design

C. Hartz, A. Mazurek, M. Miki, T. Zegard, T. Mitchell and W. F. Baker

Hangai Prize Papers for 2018

Elasto-Plastic Damper Optimization Routine for Lattice Towers

Based on Generalized Response Spectrum Analysis

Y. Terazawa and T. Takeuchi

Innovative CAD-integrated Isogeometric Simulation of Sliding Edge Cables in Lightweight Structures A. M. Bauer, R. Wüchner and K.-U. Bletzinger

Construction and History of Fuller's Timber Dome at Woods Hole J. Swerdlin, R. Mohr, P. Mayencourt, A. Brose and J. Ochsendorf

Design Strategies of Hybrid Bending-Active Systems Based on Graphic Statics and a Constrained Force Density Method L. Boulic and J. Schwartz

Technical Papers

Form Finding and Dimensioning of Reinforced Concrete Shell Roof for Akrotiri (Santorini) S. Hawdon-Earl and K. D. Tsavdaridis

A Re- Parameterization Approach for the Construction of Domes with Planar Facets R. Mesnil

Reviewers of Papers, 2018

COVER: Figures from paper by C. Hartz, A. Mazurek, M. Miki, T. Zegard, T. Mitchell and W. F. Baker

IASS Secretariat: CEDEX-Laboratorio Central de Estructuras y Materiales Alfonso XII, 3; 28014 Madrid, Spain

Tel: 3491 3357491; Fax: 3491 3357422; iass@cedex.es; http://www.iass-structures.org Printed by SODEGRAF ISSN: 1028-365X Depósito legal: M. 1444-1960 


\section{A RE- PARAMETERIZATION APPROACH FOR THE CONSTRUCTION OF DOMES WITH PLANAR FACETS}

Romain MESNIL

Researcher, Laboratoire Navier, Ecole des Ponts, IFSTTAR, CNRS, UPE, Champs-sur-Marne, romain.mesnil@enpc.fr

Editor's Note: This Manuscript submitted 18 February 2018; revision received 6 August 2018; accepted 13 September. This paper is open for written discussion, which should be submitted to the IASS Secretariat no later than June 2019.

DOI: https://doi.org/10.20898/j.iass.2018.198.016

\section{ABSTRACT}

The aim of this article is to propose parameterization with planar facets of dome structures. The technique introduced in this paper starts from an input parameterization and creates a dual pattern with planar quadrilateral facets. The derivation of the analytical solution allows to link the method with the creation of meshes with planar hexagonal facets and of circle packing on spheres. The method can be used in various contexts and allows designers to design with two superimposed parameterizations, which allows for a potential decoupling between structure and envelope.

Keywords: Structural Morphology, Constructive geometry, Fabrication-aware design, PQ-Mesh, gridshell

\section{INTRODUCTION}

Free-form structures are characterized by an interrelation between mechanical behavior, fabrication process and material. The purpose of structural morphology is to study these relations between different fields. Along the centuries, the importance of shape parameterization in architecture has been demonstrated. For example, the French mathematician Gaspard Monge proposed to cut blocks along the lines of curvature of surfaces, because the normal surface along them is developable and thus simpler to construct [16]. Recent developments in 'fabrication-aware design' follow the path of the 'founder of constructive geometry', and advocate to use specific parameterizations of surfaces to ease their fabrication. This was the case of Jörg Schlaich, Hans Schober and their team who came up with surfaces of translation and later scale-trans surfaces to construct steel glazed gridshells covered with planar facets [17]. Later, Liu et al. recalled the interest of lines of curvature for this purpose [8]. The problem of covering free-form structures with planar facets is of practical importance, numerous contributions aim at proposing efficient ways to generate so-called conjugate parameterizations of complex shapes [12].

Parameterization is also key to the understanding of numerous optimization problems in structural mechanics. For example, the solution to the famous Michell truss problem is the construction of an orthogonal parameterization known as HenckyPrandtl net [19].

Some engineers succeed in merging construction, aesthetics and structural efficiency. Hans Schober and Jörg Schlaich succeeded in constructing 'transparent shells', both lightweight and constructible [18]. Chris Williams helped structural engineers Buro Happold and architects Foster+Partners to define the geometry of the gridshell covering the great court of the British Museum with the dynamic relaxation method. The underlying principles used for the parameterization are detailed in [23]. These recent realizations of gridshells tend to merge structure and envelope because of the transparency of glass.

The aim of this paper is to study the well-known question of quadrilateral mesh planarization, while considering two superimposed patterns. Namely, we investigate the possibility to construct a planar quadrilateral mesh that is dual to a given parameterization of a surface. This allows to use one pattern as a structural pattern and the other as an envelope pattern. We put emphasis on patterns derived from principal curvature parameterization and principal stress directions, which are now wellknown in the literature on free-form architecture. 
We show how to construct a diagonal parameterization on surfaces with positive Gaussian curvature. The practical implications of using the constructed parameterization is discussed with several examples: surfaces of revolution, sphere, moulding surfaces, etc.

The second section recalls properties of conjugate networks and proposes the construction of a conjugate parameterization dual to a reference parameterization. The properties of the constructed parameterization and its relation to remarkable surface discretization are discussed in section 3 . Some possible application are shown in the fourth section.

\section{GEOMETRY OF PLANAR NETWORKS}

We start the discussion by recalling some notions of differential geometry and find the theoretical solution for the problem of surface reparameterization for planar quadrilateral facets.

\subsection{Preliminary statements}

In the followings, we consider a surface which is parameterized by its lines of curvatures.

$$
\boldsymbol{f}(u, v)=\left(\begin{array}{l}
x(u, v) \\
y(u, v) \\
z(u, v)
\end{array}\right)
$$

In which $\mathrm{u}$ and $\mathrm{v}$ are parameters and $\mathbf{f}$ is a position vector.

Several notations coexist in the literature on differential geometry, as discussed by Chris Williams in [23], who sets preference on tensor calculus in his theoretical discussion on structural parameterization of surfaces. Tensor calculus allows for concise descriptions of the variations of the different quantities related to surfaces. Green and Zerna used such notations in their reference book on elasticity [5]. Although powerful, this notation is not common for structural engineers, and since this paper does not propose a structural assessment of created patterns, we chose simple notations that do not cover shell theory, similarly to Struik [19]. A letter in subscript indicate partial derivation: for example $\boldsymbol{f}_{\boldsymbol{u}}=\frac{\partial \boldsymbol{f}}{\partial u}$

Lines of curvatures are perpendicular, so that:

$$
\boldsymbol{f}_{\boldsymbol{u}} \cdot \boldsymbol{f}_{\boldsymbol{v}}=0
$$

Moreover,

$$
f_{u v} \cdot n=0
$$

\subsection{Construction of a dual planar parameterization}

Planar quadrilateral meshes are discrete objects derived from conjugate curve networks. They are defined by a non-linear differential equation [21]:

$$
\operatorname{det}\left(\boldsymbol{f}_{X}, \boldsymbol{f}_{Y}, \boldsymbol{f}_{X Y}\right)=0
$$

Or, more simply put, a parameterization $(\mathrm{X}, \mathrm{Y})$ is a conjugate parameterization if the second crossderivative is in the osculating plane of the surface

$$
\boldsymbol{f}_{X Y} \cdot \boldsymbol{n}=0
$$

We aim here at constructing such a parameterization, which is locally symmetrical with respect to the curvature line parameterization. It is represented in Figure 1 and described by equation (6).The name 'duality' has many interpretations, and it is not used in the mathematical sense. The idea of creating a conjugate parameterization that is 'dual' to another parameterization can be found [3], where the parameterization by the lines of curvatures is dual to a Tchebycheff nets, which allows for the covering of elastic gridshells by planar facets.

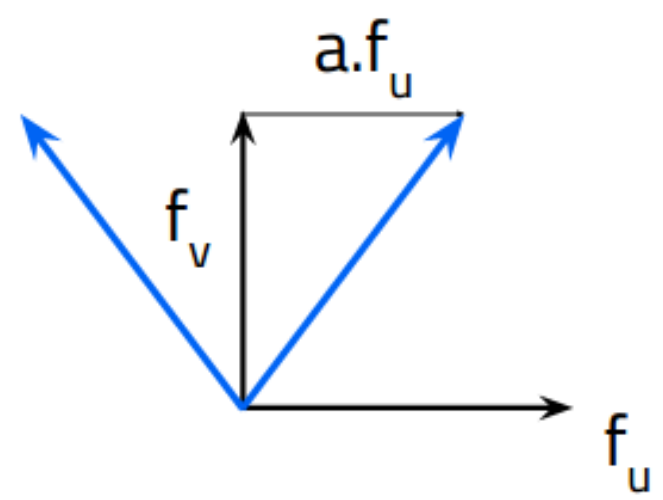

Figure 1: Diagonal parameterization constructed from an initial parameterization

$$
\left\{\begin{array}{c}
\boldsymbol{f}_{\boldsymbol{X}}=u_{X} \boldsymbol{f}_{\boldsymbol{u}}+v_{X} \boldsymbol{f}_{\boldsymbol{v}} \\
\boldsymbol{f}_{\boldsymbol{Y}}=u_{Y} \boldsymbol{f}_{\boldsymbol{u}}+v_{Y} \boldsymbol{f}_{\boldsymbol{v}} \\
\frac{u_{X}}{v_{X}}=-\frac{u_{Y}}{v_{Y}}=a(u, v)
\end{array}\right.
$$


The cross derivative is simply computed as follows:

$$
\boldsymbol{f}_{X Y}=u_{X Y} \boldsymbol{f}_{\boldsymbol{u}}+u_{X} u_{Y} \boldsymbol{f}_{\boldsymbol{u} \boldsymbol{u}}+\left(u_{X} v_{Y}+u_{Y} v_{X}\right) \boldsymbol{f}_{\boldsymbol{u} v}+
$$

We can thus evaluate the component along the normal of the surface $\boldsymbol{n}$, defined as $\boldsymbol{n}=\boldsymbol{f}_{\boldsymbol{u}} \wedge \boldsymbol{f}_{\boldsymbol{v}}$.

$$
\boldsymbol{f}_{X Y} \cdot \boldsymbol{n}=u_{X} u_{Y} \boldsymbol{f}_{\boldsymbol{u} \boldsymbol{u}} \cdot \boldsymbol{n}+\left(u_{X} v_{Y}+u_{Y} v_{X}\right) \boldsymbol{f}_{\boldsymbol{u} \cdot} \cdot \boldsymbol{n +}
$$

This expression can be simplified when $(\mathrm{u}, \mathrm{v})$ is a curvature line parameterization and satisfies equation (3):

$$
\boldsymbol{f}_{X Y} \cdot \boldsymbol{n}=u_{X} u_{Y} \boldsymbol{f}_{\boldsymbol{u} \boldsymbol{u}} \cdot \boldsymbol{n}+v_{X} v_{Y} \boldsymbol{f}_{\boldsymbol{v} \boldsymbol{v}} \cdot \boldsymbol{n}
$$

Since we are looking for conjugate parameterizations, this quantity must satisfy equation (5). This imposes a restriction on the value of $a$ :

$$
-a^{2} \boldsymbol{f}_{u u} \cdot \boldsymbol{n}+\boldsymbol{f}_{v v} \cdot \boldsymbol{n}=\mathbf{0}
$$

Assuming that $(\mathrm{u}, \mathrm{v})$ is a curvature line parameterization, we can express the quantities $\boldsymbol{f}_{\boldsymbol{u} \boldsymbol{u}} \cdot \boldsymbol{n}$ and $\boldsymbol{f}_{\boldsymbol{v} \boldsymbol{v}} \cdot \boldsymbol{n}$ with the principal curvatures $\kappa_{u}$ and $\kappa_{v}$. First, we notice that equation (10) is well posed only if the surface has positive Gaussian curvature. It follows that

$$
a=\frac{\left\|\boldsymbol{f}_{v}\right\|}{\left\|\boldsymbol{f}_{u}\right\|} \sqrt{\frac{\kappa_{v}}{\kappa_{u}}}
$$

\section{DISCUSSION}

\subsection{Link with Dupin's indicatrix and planar hexagonal meshes}

Wang et al. showed that vertices of a hexagonal mesh with planar facets converge towards Dupin indicatrix of the underlying surface [25]. Our result shows a remarkable relation with this construction, which is illustrated in Figure 2.

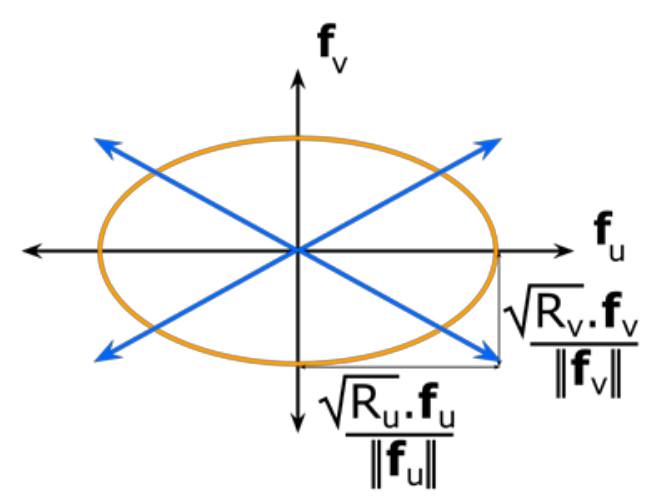

Figure 2: Dupin indicatrix as an ellipse: principal curvature direction (black) and the parameterization constructed in this paper (blue)

Indeed, in positive curvature area, Dupin's indicatrix is an ellipse, and the ratio of the semiaxes lengths is:

$$
\frac{l_{u}}{l_{v}}=\frac{\sqrt{R_{u}}}{\sqrt{R_{v}}}
$$

In the (u,v) coordinates, one must take into account that $\boldsymbol{f}_{\boldsymbol{u}}$ and $\boldsymbol{f}_{\boldsymbol{u}}$ are not unitary. This is equal to the ratio $a$ computed previously in equation (11).

In planar hexagonal meshes, one is able to naturally extract three families of developable strips, which are highlighted in Figure 3. One family of strips is aligned with a principal curvature direction, while the two others are aligned with the parameterization constructed in this article. Regular planar hexagonal meshes are notably difficult to obtain because one must control the metric of the parameterization.

Our considered parameterization cannot be constructed for surfaces with negative Gaussian curvature. It should be noticed that for surfaces of negative Gaussian curvature, the Dupin's indicatrix is a hyperbola, so that the resulting strips are not smooth. 

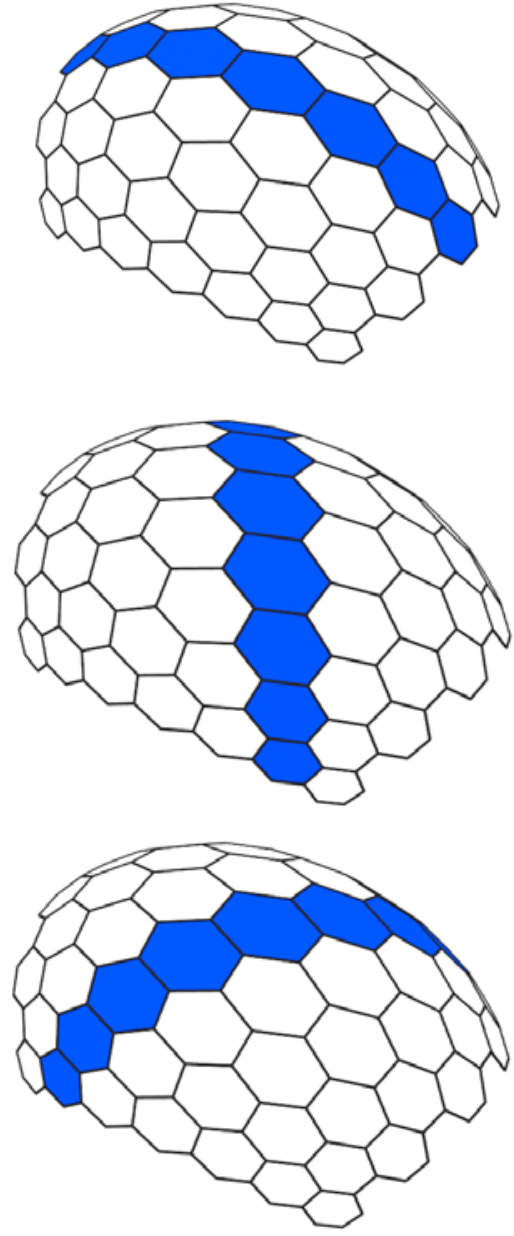

Figure 3: A hexagonal mesh with planar facets: alignment of the mesh with principal curvature (top) and the dual parameterization constructed in this paper (center and bottom)

Finally, it can be noticed that the parameterization constructed in equation (11) corresponds to the imaginary asymptotes of the ellipse [22]. It is interesting to recall that the asymptotic directions in surfaces with negative Gaussian curvature are selfconjugated: and cannot be used to construct quadrilateral meshes with planar facets [8]. A fundamental difference between surfaces of positive or negative curvature is thus illustrated by our construction.

\subsection{Surfaces of revolution}

The lines of curvature of surfaces of revolution are the meridians and parallels. They are therefore extremely simple to characterize from an analytical point of view and we propose here to study the properties of the diagonal parameterization constructed in this paper.

$$
\cos \alpha=\sqrt{\frac{\kappa_{v}}{\kappa_{u}}}
$$

The curvatures $\kappa_{u}$ and $\kappa_{v}$ remain constant along parallels, the angle of the parameterization is thus also constant. This guarantees already good repetition of elements in the constructed parameterization. We propose to find surfaces so that the angle does not vary along the parallels either. Such parameterizations are known as loxodromic parameterizations: the loxodromic, also called rhumb lines, make a constant angle with the meridians.

We consider a surface of revolution with following cylindrical equation:

$$
\left\{\begin{array}{c}
x(t, \varphi)=\rho(t) \cos \varphi \\
y(t, \varphi)=\rho(t) \sin \varphi \\
z(t, \varphi)=h(t)
\end{array}\right.
$$

In the followings, we assume that $\rho_{t}{ }^{2}+h_{t}{ }^{2}=1$ and compute the couple $(\rho, h)$ so that $\sqrt{\frac{\kappa_{\varphi}}{\kappa_{t}}}=\beta$. The computation of the principal curvatures is classical [7] and yields a couple of nonlinear equations.

$$
\left\{\begin{array}{c}
\rho . \rho_{t t}+\beta\left(1-\rho_{t}{ }^{2}\right)=0 \\
\rho_{t}{ }^{2}+h_{t}{ }^{2}=1
\end{array}\right.
$$

The first equation in (13) depends on $\rho$ only. Its solution is not expressed with classical functions for general values of $\beta$. We shall notice however that there is an obvious solution for $\beta=1$ :

$$
\left\{\begin{array}{l}
\rho=\cos t \\
h=\sin t
\end{array}\right.
$$

We find thus the well-known parameterization of the sphere. In the case of a sphere, the principal curvatures are indeed a constant and $\cos \alpha=1$, so that the two networks of the diagonal parameterization make a constant angle of $90^{\circ}$. This theoretical solution has been applied on a notable example: the Maritime Museum of Osaka designed by Paul Andreu, Tohata Architects and Ove Arup \& Partners. We shall notice however that the designers constructed the pattern with another rule and were not certain that the spiraling curves were loxodromic curves [6]. 


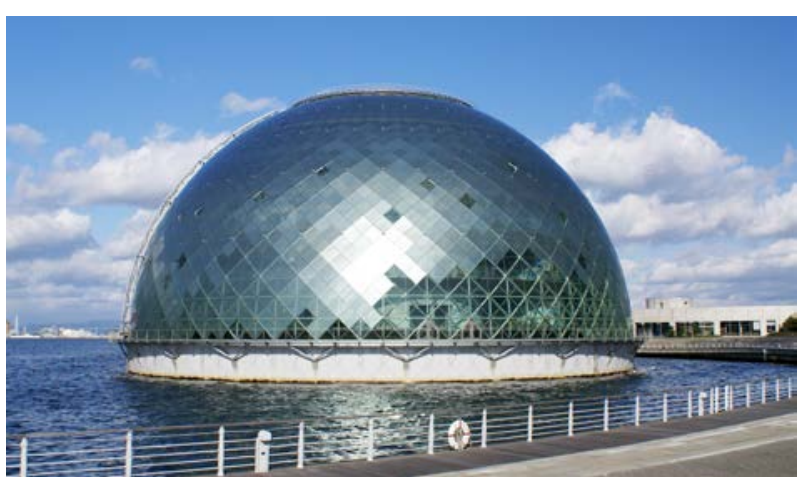

Figure 4: Osaka Maritime Museum: a spiraling pattern following loxodromic curves and creating a PQ-Mesh

Other values of $\beta$ can be used, yielding different shapes. The numerical solution of equation (13) is found with the integration of a finite difference scheme detailed in the appendix. Some profile curves are represented in Figure 5. Large values of $\beta$ correspond to high rise-over-span ratio whereas small values of $\beta$ result in shallow profile curves.

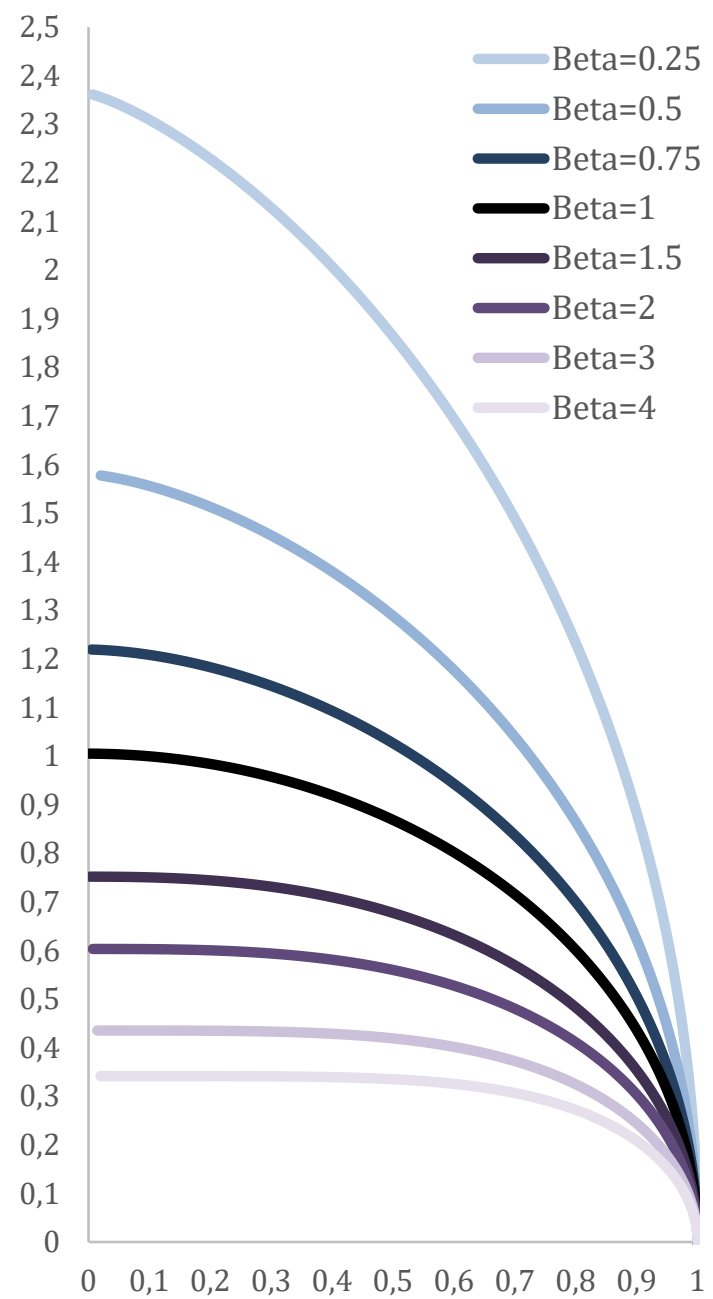

Figure 5: Profile curve of surfaces of revolution where loxodromic curves create a conjugate curve network

\subsection{Sphere meshing}

We consider now patterns derived from lines of curvature on spheres. Equation (11) can be simplified in the case of a sphere:

$$
\left\{\begin{array}{c}
\boldsymbol{f}_{X}=\left\|\boldsymbol{f}_{v}\right\| \cdot \boldsymbol{f}_{\boldsymbol{u}}+\left\|\boldsymbol{f}_{\boldsymbol{u}}\right\| \cdot \boldsymbol{f}_{v} \\
\boldsymbol{f}_{Y}=-\left\|\boldsymbol{f}_{v}\right\| \cdot \boldsymbol{f}_{\boldsymbol{u}}+\left\|\boldsymbol{f}_{\boldsymbol{u}}\right\| \cdot \boldsymbol{f}_{v}
\end{array}\right.
$$

This can be written as follows:

$$
\left\{\begin{array}{c}
f_{X}=f_{U}+f_{V} \\
f_{Y}=-f_{U}+f_{V}
\end{array}\right.
$$

with

$$
\left\{\begin{array}{l}
\boldsymbol{f}_{U}=\left\|\boldsymbol{f}_{v}\right\| \cdot \boldsymbol{f}_{\boldsymbol{u}} \\
\boldsymbol{f}_{\boldsymbol{V}}=\left\|\boldsymbol{f}_{u}\right\| \cdot \boldsymbol{f}_{v}
\end{array}\right.
$$

The parameterization $(\mathrm{U}, \mathrm{V})$ is isothermic because it satisfies two identities:

$$
\left\{\begin{array}{c}
\left\|f_{U}\right\|=\left\|f_{V}\right\| \\
f_{U} \cdot f_{V}=0
\end{array}\right.
$$

This construction admits a discrete counterpart, already discussed by Bobenko and Springborn [2]. Isothermic parameterizations of the sphere can indeed be constructed from circle packings. The top of Figure 6 shows a circle packing on a sphere: connected circles a touching tangentially at one point. A mesh can be constructed so that the circles are in-circles of the facets: it corresponds to a discrete isothermic parameterization of the sphere. Another circle packing, dual to the first one can be constructed so that the circles of the two circle packing are perpendicular and share the same contact points between circles. This second circle packing is shown in light orange in Figure 6. From the two circle packing, one can create a circular mesh, with the vertices of all facets inscribed in a circle.

This remark further demonstrates that the dual parameterization introduced in this article is constrained in practice. For a given pattern connectivity, the circle packing is indeed unique up to a Möbius transformation. 

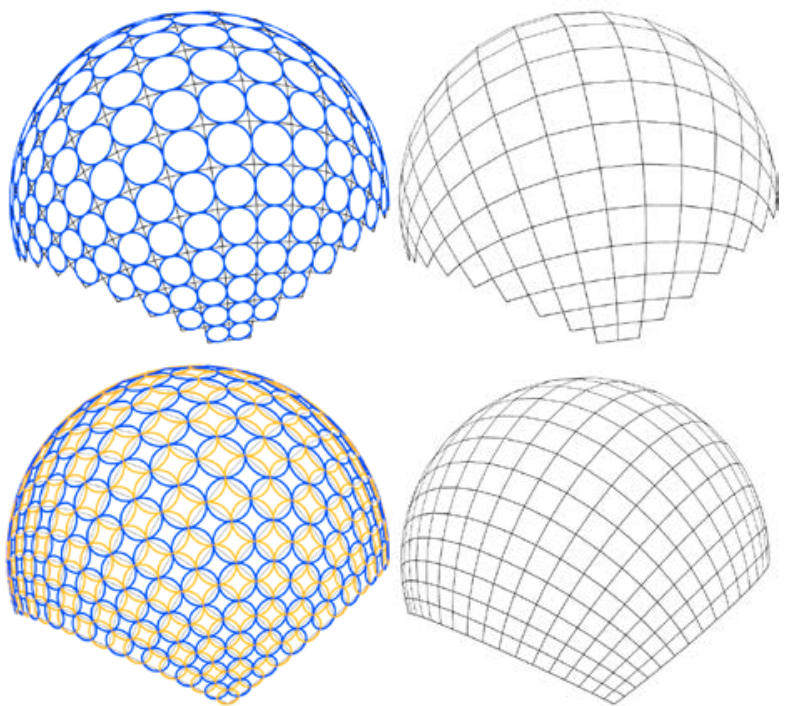

Figure 6: Circle packing on a sphere: isothermic parameterization (top) and corresponding dual parameterization derived in this article (bottom)

\section{APPLICATIONS}

\subsection{Implementation}

The examples presented in the present paper are computed on NURBS surfaces, where the computation of the derivatives is exact and fast. We use the classical Runge-Kutta method for the vector field integration.

It is also possible to perform vector field integration on meshes with methods already existing in the literature [9].

\subsection{Gridshells with high node congruence}

High node repetition can be obtained by applying parallel transformations to meshes with node congruence, because such transformations leave the geometry of nodes invariant. This principle has been applied to the networks of lines of curvature in [10]. It can be extended to the loxodromic parameterization of surfaces of revolutions proposed in this paper.

\subsection{Pattern superposition}

The parameterization proposed in this article offers the potential to work with two different patterns for the structure and envelope. The curve network following the parameterization satisfying equation (10) have their intersection at constant values of $u$ and v. Consider Figure 7: the blue lines represent the conjugate parameterization constructed from equation (9) while the black dotted lines represent isocurves of the initial parameterization. The blue lines intersect along the iso-curves (solid circles): the white circles indicates that only a subset of the isocurve network is used.

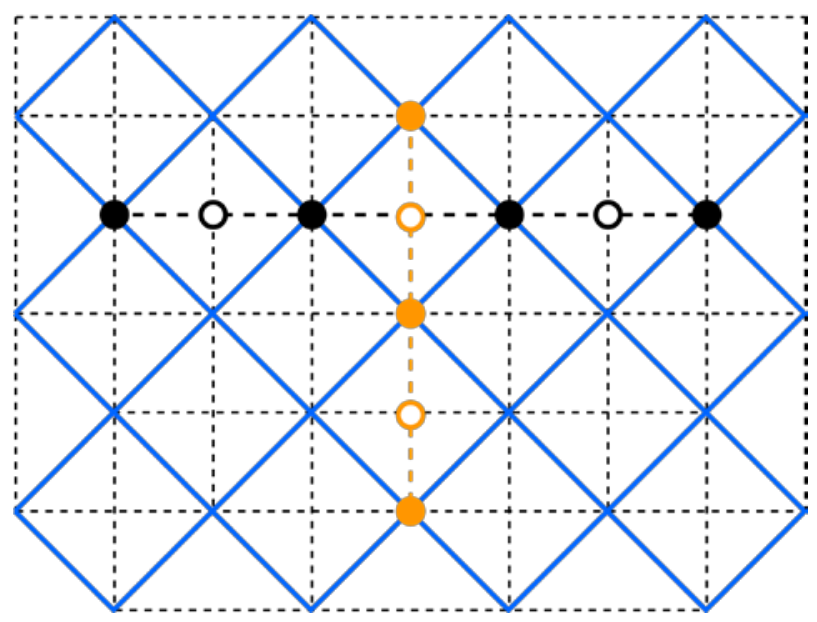

Figure 7: Two superposed parameterization: the vertices of the blue and black dashed patterns are in common

The literature on free-form structures does not offer many examples of pattern superposition strategies, one of the only ones being the gridshells of the Paunsdorf Center, which uses a triangular structural pattern and a quadrangular pattern for glazing, as shown in Figure 8 [18]. Triangular gridshells are known to be significantly stiffer than quadrangular gridshells, and quadrangles have better nesting properties than triangles, so this solution uses the advantages of both solutions.

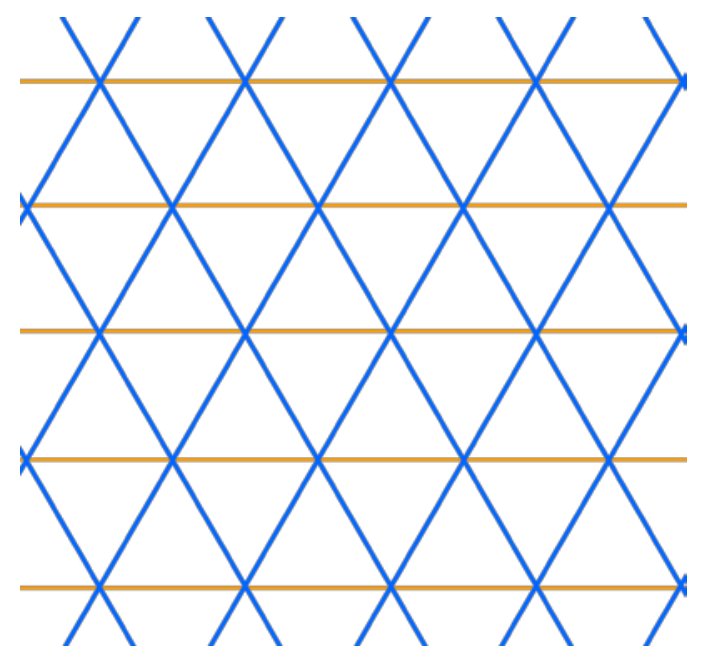

Figure 8: Patterns used for the Paunsdorf gridshell: the glazing pattern (blue) and structural pattern (orange and blue) 
Our approach allows to use different strategies for patterning, for example if the structural pattern follows structurally optimal load paths. It can also be extended to other patterning strategies, like the Kagome pattern. Indeed, Kagome gridshells have better performance than quadrangular patterns, with the same node valency [13][14]. The nesting of triangles and hexagons is however not as efficient as the nesting of quadrilaterals, so exploring the same idea as the one developed for the Paunsdorf Center gridshell is a possibility offered by our approach.

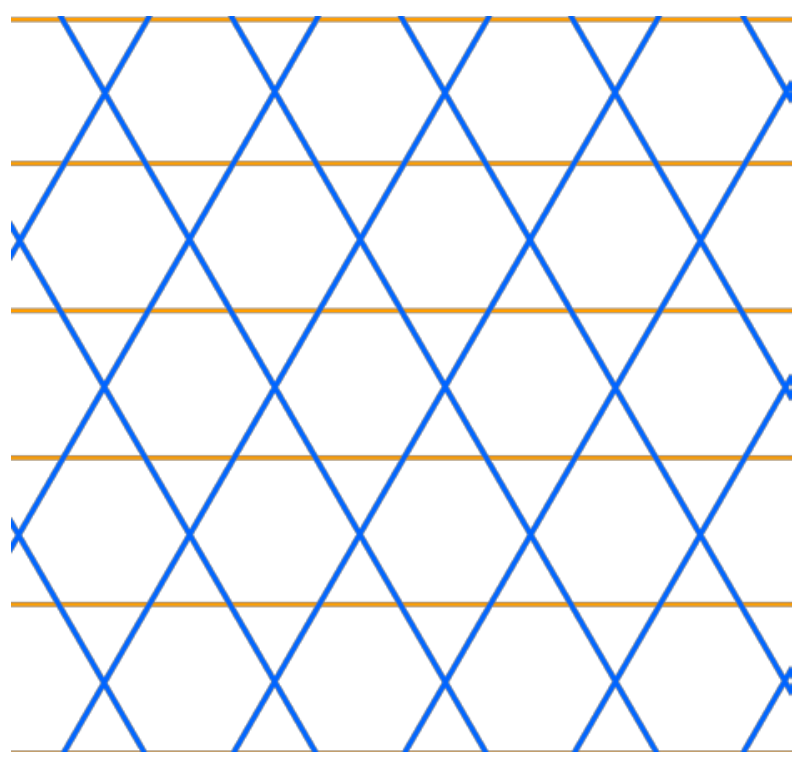

Figure 9: A kagome grid pattern: envelope (blue) and structure (blue and orange)

Notice however that the pattern superposition imposes strong constraints on the metric because of equation (10). This is obvious with the sphere, where the initial pattern must derive from an isothermic parameterization. The designer loses thus degrees of freedom on the subdivision of the initial mesh.

\subsection{Structurally optimal patterns}

Structurally optimal pattern in plates are known to follow lines of principal stress. When a load case is dominant, it is meaningful to take inspiration from the principal stress orientation to derive a structural pattern, as illustrated by ribbed slabs designed by Pier Luigi Nervi [1]. This approach is however difficult to generalize to lightweight structures, where the self-weight is not the governing load for the structural design.
Remarkably, lines of curvature are also lines of principal stress for uniform normal load for a family of surfaces, for example isothermic surfaces [15]. Isothermic surfaces cover a large range of shapes, like minimal surfaces, constant mean-curvature surfaces, quadrics, like the ellipsoid. Gaspard Monge illustrated the potential of his findings by proposing the design of a vault with the blocks aligned with the principal curvatures of an ellipsoid. This was a computational tour de force at that time, and the result was highly aesthetical. We propose to re-investigate the patterning of ellipsoids with the method proposed in this article. The result can be seen in Figure 10 and Figure 11.

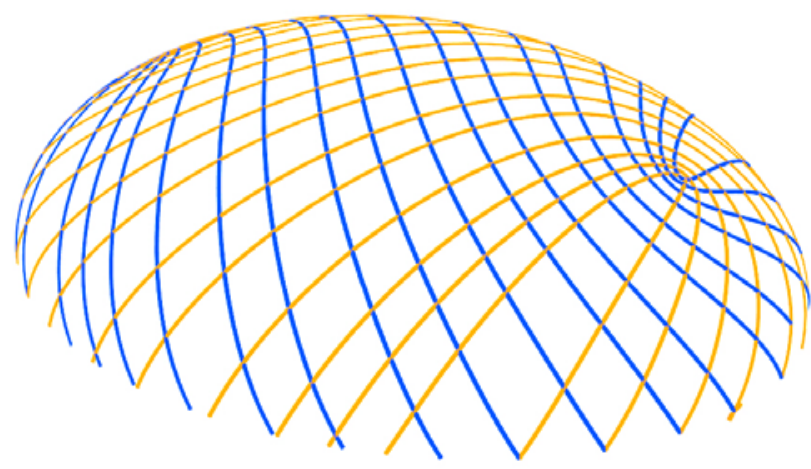

Figure 10: Conjugate curve network on an ellipsoid constructed with our method

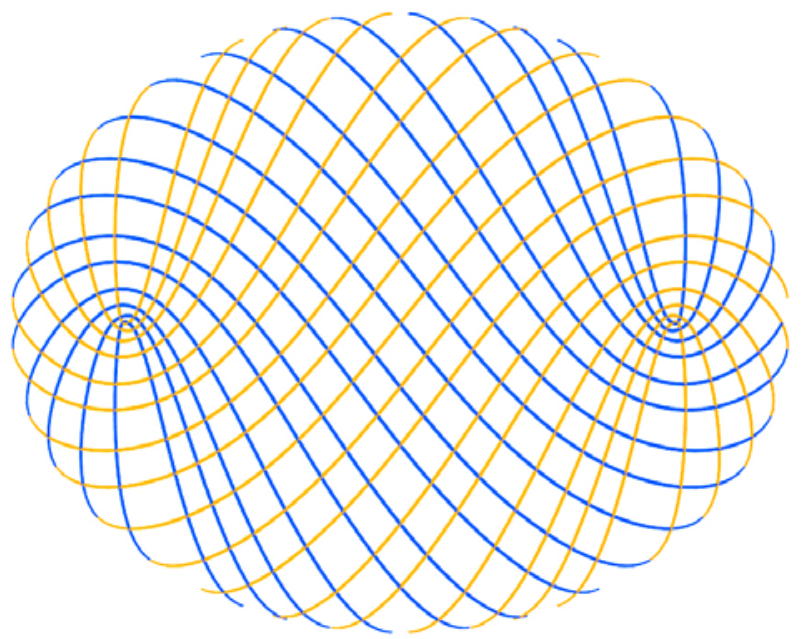

Figure 11: Conjugate curve network dual to the lines of curvature on an ellipsoid

The pattern superposition approach offers thus a potential for the creation of efficient structures with elegant parameterizations. 


\section{5. $\quad$ PQ-meshes and developable strips}

Figure 12 shows a stadium geometry based on a moulding surface. Moulding surfaces are generated by the sweeping of a planar curve along a planar rail curve. Alternatively, they can be viewed as images of surfaces of revolution by parallel transformations [10]. Their lines of curvature are planar, which simplifies their assembly and construction. Moulding surfaces are regularly used for the geometric generation of stadia.

The pattern showed in Figure 12 is dual to lines of curvatures. It breaks the reading of these planar curves, and highlights the visual potential of the spiraling patterns arising from our reparameterization approach. Note that similar looking diagrid patterns have been used in recent stadia projects, like the Arena da Amazônia, built in Manaus, Brazil in 2014[4]. In this project, the diagrid behaves like a gridshell, and is stabilized by a compression ring at the inner edge and by a tension ring at the outer edge. Therefore, the alignment of the pattern with the edges of the surface is a necessary condition to guarantee a proper structural behavior, so that the approach proposed in this article could be used for this purpose.

Finally, the Arena da Amazônia was covered with a PTFE membrane: the method proposed in this article could extend the range of materials or technologies for covering, for example with developable panels which could fulfil a structural purpose as a bracing system. In Figure 12, one of the developable surface created by the parameterization is shown in light blue, while a developable surface resulting from the curvature line parameterization is depicted in light orange.

\section{CONCLUSION}

This article introduced a re-parameterization technique on curved shapes that yields planar quadrilateral facets. The main advantage of the method is that it allows the superposition of a structural pattern and an envelope pattern. The construction of the solution is linked with classical objects of discrete geometry: circle packing on spheres and hexagons.
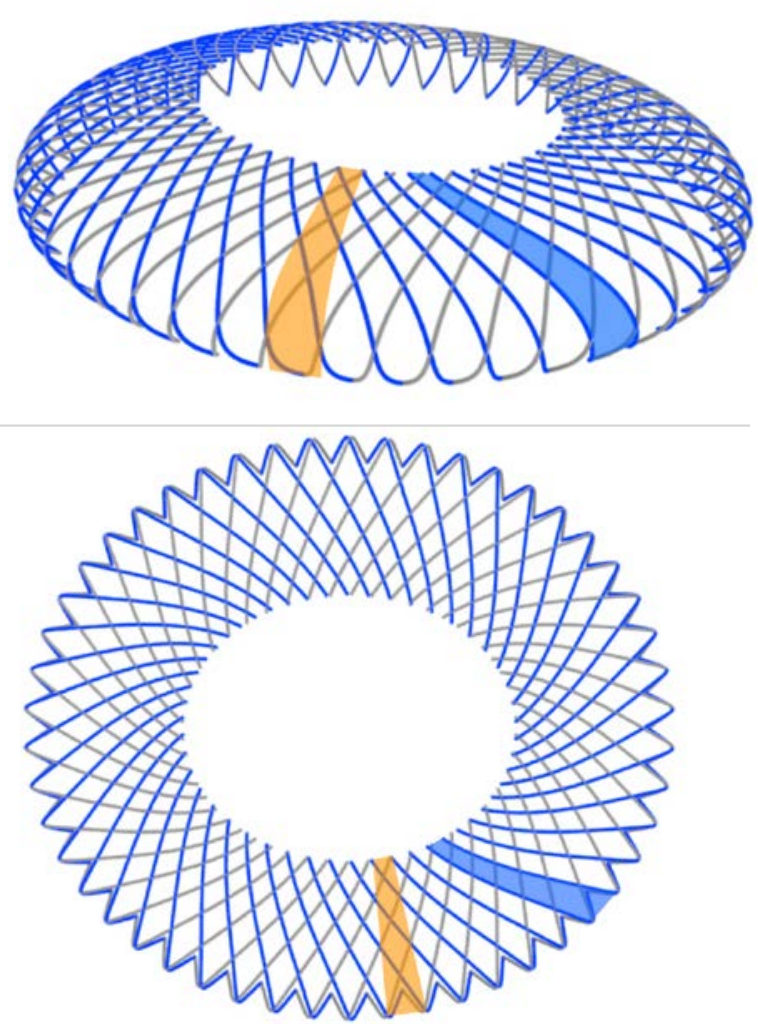

Figure 12: A stadium geometry based on a moulding surface: perspective (top) and plane view (bottom). The surface between two consecutive curves of the same color is developable (shown in blue)

We described the surfaces which admit a conjugate curve parameterization by its rhumb lines with a system of nonlinear equations. The analytical solution has been derived for specific cases, and a discrete integration scheme has been proposed. The resulting structures have a constant angle and planar facets.

The proposed method allows the designer to manipulate and combine two parameterizations. This allows the separation of structural pattern and envelope pattern and should open new possibilities for the structural design of domes.

\section{ACKNOWLEDGMENTS}

The author would like to thank Professor Nicolas Pauli and Professor Ken'ichi Kawaguchi for remarks and discussions that led to the idea behind this paper. 


\section{PHOTO CREDITS}

Figure 4 is licensed under the Creative Commons Attribution 2.5 Generic license. Complementary informations can be found on this link:

https://commons.wikimedia.org/wiki/File:Osaka_m aritime_museum01s3200.jpg

\section{REFERENCES}

[1] Billington, D. P. The tower and the bridge: the new art of structural engineering. Princeton University Press, 1985. ISBN: 069102393X.

[2] Bobenko, A., and Boris Springborn, B. "Variational principles for circle patterns and Koebe's theorem". Transactions of the American Mathematical Society, 356, 2, 659689, 2004. (DOI: 10.1090/S0002-9947-0303239-2)

[3] Douthe, C., Mesnil, R., Orts, H., \& Baverel, O. "Isoradial meshes: Covering elastic gridshells with planar facets". Automation in Construction, 83, 222-236, 2017. (DOI: 10.1016/j.autcon.2017.08.015)

[4] Göppert, K., Stockhusen K., \& Stein, M. "Brazilian trio". Civil Engineering Magazine Archive 84(11), 80-92, 2014. (DOI: 10.1061/ciegag.0000515)

[5] Green, A. E., and Zerna, W. Theoretical elasticity. Courier Corporation, 1992. ISBN: 0486670767.

[6] Kazuo, K., Oguri, A., \& Sasaki, J. “Osaka Maritime Museum, Japan”. Concrete, 36(7), 12-13, 2002.

[7] Kühnel, W. "Differential geometry: curvessurface-manifolds". American Mathematical Society, 75-82, 2002. ISBN: 978-1-47042320-9.

[8] Liu, Y., and Pottmann, H., and Wallner, J., and Yang, Y. L., and Wang, W. "Geometric modeling with conical meshes and developable surfaces". ACM Transactions on Graphics (TOG). 25(3), 2006. (DOI: 10.1145/1141911.1141941)
[9] Liu, Y., et al. "General planar quadrilateral mesh design using conjugate direction field". ACM Transactions on Graphics (TOG), 30(6), 2011.

(DOI: 10.1145/2070781.2024174)

[10] Mesnil, R. and Douthe, C. and Baverel, O. and Léger, B. and Caron, J.-F. "Isogonal moulding surfaces: a family of shapes for high node congruence in free-form structures”. Automation in Construction, 59, 38-47, 2015.

(DOI: 10.1016/j.autcon.2015.07.009)

[11] Mesnil, R. and Douthe, C. and Baverel, O. and Léger, B. "Generalised cyclidic nets for shape modelling in architecture". International Journal of Architectural Computing, 15(2) 148-168, 2017. (DOI: 10.1177/1478077117714917)

[12] Mesnil, R., and Douthe, C., and Baverel, O., and Léger, B. "Marionette Meshes: modelling free-form architecture with planar facets". International Journal of Space Structures. 32(3-4), 184-198, 2017. (DOI: $10.1177 / 0266351117738379)$

[13] Mesnil, R., and Douthe, C., and Baverel, O., and Léger, B. "Linear buckling of quadrangular and kagome gridshells: a comparative assessment". Engineering Structures, 132, 337-348, 2017. (DOI: 10.1016/j.engstruct.2016.11.039)

[14] Mesnil, R., and Douthe, C., and Baverel, O. "Non-standard patterns for gridshells: fabrication and structural optimization". Journal of the International Association for Shell and Spatial Structures. 58(4), 277-286, 2017. (DOI: 10.20898/j.iass.2017.194.893)

[15] Rogers, C., \& Schief, W. K. "On the equilibrium of shell membranes under normal loading. Hidden integrability". In Proceedings of the Royal Society of London A: Mathematical, Physical and Engineering Sciences, 459, 2038, 2449-2462, 2003. (DOI: 10.1098/rspa.2003.1135)

[16] Sakarovitch, J. "Gaspard Monge Founder of 'Constructive Geometry'." Proceedings of the Third International Congress on Construction History. 2009. URL: 
https://halshs.archives-ouvertes.fr/hal00712704/

[17] Schlaich, J., \& Schober, H. "Glass-covered grid-shells”. Structural engineering international. 6(2), 88-90, 1996. (DOI: $10.2749 / 101686696780495716)$

[18] Schober, H. Transparent shells: Form, topology, structure. John Wiley \& Sons, 2015. ISBN: 9783433031216.

[19] Strang G., Kohn R. V. "Hencky-Prandtl nets and constrained Michell trusses" Computer Methods in Applied Mechanics and Engineering. 36(2), 207-222, 1983. (DOI: 10.1016/0045-7825(83)90113-5)

[20] Struik, D. J. Lectures on Classical Differential Geometry. 2nd ed. New York: Dover Publications, 1988. ISBN: 9780486656090 .

[21] Suris, Y. B., \& Bobenko, A. I. Discrete differential geometry: Integrable structure, American Mathematical Society, 2008. ISBN: 0821847007.

[22] Vaisman, I. Analytical geometry. Vol. 8. World Scientific, pp. 115-116, 1997. ISBN: 978-981-02-3158-3.

[23] Williams, C.J.K. The analytic and numerical definition of the geometry of the British Museum Great Court Roof, 434-440, 2001. ISBN: 0-7300-2526-8.

[24] Williams, C.J.K. "Patterns on a Surface: The Reconciliation of the Circle and the Square". Nexus Network Journal. 13(2), 281-295, 2011. (DOI: 10.1007/s00004-011-0068-2)

[25] Wang, W., Liu, Y., Yan, D., Chan, B., Ling, R., \& Sun, F. Hexagonal meshes with planar faces. Dept. of CS, HKU, Tech. Rep., 2008.

\section{APPENDIX 1: INTEGRATION OF} LOXODROMIC

Equation (12) cannot be solved using usual functions. We implement thus a finite difference scheme for its integration. We use the central finite difference,

$$
\left\{\begin{array}{c}
\rho_{t t}(t)=\frac{\rho(t+\Delta t)-2 \cdot \rho(t)+\rho(t-\Delta t)}{\Delta t}+\mathcal{O}\left(\Delta t^{2}\right) \\
\rho_{t}(t)=\frac{\rho(t+\Delta t)-\rho(t-\Delta t)}{2 \Delta t}+\mathcal{O}\left(\Delta t^{2}\right)
\end{array}\right.
$$

We write $\rho(t+\Delta t)=\rho^{+}$and $\rho(t-\Delta t)=\rho^{-}$, so that equation (13) becomes:

$$
\rho \cdot \frac{\rho^{+}-2 \rho+\rho^{-}}{\Delta t}+\beta\left(1-\frac{\left(\rho^{+}-\rho^{-}\right)^{2}}{4 \Delta t^{2}}\right)+\mathcal{O}\left(\Delta t^{2}\right)=0
$$

Assume that one knows $\rho^{-}$and $\rho$ : the equation is simply a second order equation in $\rho^{+}$and has an obvious solution. For the initialization, we assume that $\rho_{t}=0$, so that $\rho(\Delta t)=\rho(-\Delta t)$. The first step of the algorithm is expressed as follows:

$$
\rho \cdot \frac{2 \rho^{+}-2 \rho}{\Delta t}+\beta+\mathcal{O}\left(\Delta t^{2}\right)=0
$$

So that the solution is

$$
\rho^{+} \sim \rho-\frac{\beta \Delta t}{2 \rho}
$$

The finite difference methods allows thus to integrate the value of $\rho$. The value of $\mathrm{h}$ is found by integrating the following equation by using the central difference scheme:

$$
h_{t}=\sqrt{1-\rho_{t}^{2}}
$$

